PROCEEDINGS OF THE AMERICAN MATHEMATICAL SOCIETY

Volume 126, Number 5, May 1998, Pages 1487-1490

S 0002-9939(98)04226-9

\title{
TWIST POINTS OF THE VON KOCH SNOWFLAKE
}

\author{
FAUSTO DI BIASE, BERT FISCHER, AND RÜDIGER L. URBANKE
}

(Communicated by Albert Baernstein II)

\begin{abstract}
It is known that the set of twist points in the boundary of the von Koch snowflake domain has full harmonic measure. We provide a new, simple proof, based on the doubling property of the harmonic measure, and on the existence of an equivalent measure, invariant and ergodic with respect to the shift.
\end{abstract}

The von Koch snowflake domain $D$ is the union of an increasing sequence of open polygons $D_{n}$, where $D_{0}$ is an equilateral triangle, and $D_{n+1}$ is obtained from $D_{n}$ by replacing the middle third of each side of $D_{n}$ by the two other sides of the equilateral triangle based on the removed segment and lying outside $D_{n}[\mathrm{vK} 06]$. A point $\omega \in b D$ is called a twist point if

$$
\liminf _{z \rightarrow \omega, z \in \sigma} \arg [z-\omega]=-\infty, \quad \limsup _{z \rightarrow \omega, z \in \sigma} \arg [z-\omega]=+\infty
$$

for each curve $\sigma$ in $D$ ending at $\omega[\mathrm{P} 92, \mathrm{p} .141]$. Let $\nu_{p}$ be the harmonic measure on $b D$ for $D$ with respect to the center $p$ of $D_{0}$. We show that the following known theorem admits a new, simple proof, based on the doubling property of $\nu_{p}$ and on the existence of a measure equivalent to $\nu_{p}$, invariant and ergodic with respect to the shift.

Theorem. The set of twist points in the boundary of the von Koch snowflake has full harmonic measure.

Let $T_{n}$ be the collection of the sides of $D_{n}$. For $n=1,2, \ldots$ we now define a labeling $\ell: T_{n} \rightarrow \mathcal{A}$, where $\mathcal{A}=\left\{l, r, c_{l}, c_{r}\right\}$. For each $x \in T_{n}(n=0,1,2, \ldots)$, the left third of $x$ is an element of $T_{n+1}$ and is labeled by $l$; the right third of $x$ (also an element of $T_{n+1}$ ) is labeled by $r$; the equilateral triangle based on the middle third of $x$ has two other sides (again, elements of $T_{n+1}$ ): the one adjacent to the left (right) third is labeled by $c_{l}\left(c_{r}\right)$. The subset of $T_{n+1}$ just described is denoted by $\lambda_{+}(x)$; its elements are called direct descendants of $x$; each $y \in T_{n+1}$ is a direct descendant of a unique element of $T_{n}$, denoted by $y^{-}$. Let $T=\bigcup_{n=0}^{\infty} T_{n}$. For $x \in T$ we write $|x|=n$ if $x \in T_{n}$.

Let $C$ be the collection of the vertices of all the polygons $D_{n}$; since $C$ is countable, its harmonic measure is zero [HK76, p.247]. For each $\omega \in b D \backslash C$ there is a

Received by the editors November 1, 1996.

1991 Mathematics Subject Classification. Primary 31A15, 30C35.

Key words and phrases. Harmonic measure, von Koch snowflake, doubling property, shift, ergodicity, twist points.

The first author was supported by CNR Grants 203.01.55 and 203.01.63

The second author was partially supported by the Alexander von Humboldt Foundation.

(c)1998 American Mathematical Society 
unique sequence $\{\omega(n)\}_{n=0}^{\infty}$ in $T$ such that $\omega(n) \in T_{n}, \omega(n)=\omega(n+1)^{-}$and $\lim _{n \rightarrow \infty} \inf _{z \in \omega(n)}|z-\omega|=0$. Conversely, each sequence $\left\{x_{n}\right\}$ in $T$ such that $x_{n} \in T_{n}$ and $x_{n}=x_{n+1}^{-}$determines a unique point $\omega \in b D$ such that

$$
\lim _{n \rightarrow \infty} \inf _{z \in x_{n}}|z-\omega|=0 .
$$

For $x \in T$ let $E(x)=\{\omega \in b D: \omega(|x|)=x\}$.

Lemma 1. There is a number $\epsilon \in(0,1 / 2)$ such that for each $x \in T \backslash T_{0}$ one has

$$
\epsilon \nu_{p}\left(E\left(x^{-}\right)\right) \leq \nu_{p}(E(x)) .
$$

Proof. Observe that a fixed dilation of the Euclidean ball $E\left(x^{-}\right)$contains $E(x)$, and apply the doubling property of the harmonic measure of the snowflake.

Fix $x_{0} \in T_{0}$. Since $\nu_{p}\left(E\left(x_{0}\right)\right)=1 / 3$, it is enough to show that the set of twist points of $b D$ belonging to $E\left(x_{0}\right)$ has harmonic measure equal to $1 / 3$. Let $\Omega:=E\left(x_{0}\right) \backslash C$ and let $\nu:=3 \nu_{p}$ on $\Omega$. The labeling $\ell: T_{n} \rightarrow \mathcal{A}$ induces the embedding $J: \Omega \rightarrow \mathcal{A}^{\mathbb{N}}$ given by $\omega \in \Omega \mapsto J(\omega):=\{\ell(\omega(n))\}_{n=1}^{\infty}$. Consider the projection $\pi: \mathcal{A}^{\mathbb{Z}} \rightarrow \mathcal{A}^{\mathbb{N}}$ which maps $\left\{x_{j}\right\}_{j=-\infty}^{\infty}$ into $\left\{x_{j}\right\}_{j=1}^{\infty}$, and the shift $S: \mathcal{A}^{\mathbb{Z}} \rightarrow \mathcal{A}^{\mathbb{Z}}$ which maps a sequence $\left\{x_{j}\right\}_{j}$ into the sequence $\left\{x_{j+1}\right\}_{j}$.

Lemma 2. There is a probability measure $\mu$ on $\mathcal{A}^{\mathbb{Z}}$, invariant and ergodic with respect to $S$, and there are positive finite constants $C_{1}$ and $C_{2}$ such that for all $x \in T$ with $E(x) \subset \Omega$ one has $C_{1} \nu(E(x)) \leq \mu\left(\pi^{-1}(J(E(x)))\right) \leq C_{2} \nu(E(x))$.

Proof. See Lemma 2 in [C85], or [S94, pp.123-130].

We now show that the condition that a point $\omega \in \Omega$ is a twist point can be easily expressed in terms of the sequence $\{\omega(n)\}$; to this end, we define $\alpha\left(x_{0}\right)$ to be the number between 0 and $2 \pi$ such that $e^{i \alpha\left(x_{0}\right)}$ is the inward normal vector to $x_{0}$. Then we set

$$
\begin{array}{rll}
\alpha(x)=\alpha\left(x^{-}\right) & \text {if } & \ell(x)=l \text { or } r, \\
\alpha(x)=\alpha\left(x^{-}\right)+\pi / 3 & \text { if } \quad & \ell(x)=c_{l}, \\
\alpha(x)=\alpha\left(x^{-}\right)-\pi / 3 & \text { if } \quad & \ell(x)=c_{r},
\end{array}
$$

which guaranties that $e^{i \alpha(x)}$ is always the inward normal vector to $x$.

Lemma 3. If $\omega \in \Omega$ and

$$
\limsup _{n \rightarrow \infty} \alpha(\omega(n))=\infty \text { and } \liminf _{n \rightarrow \infty} \alpha(\omega(n))=-\infty,
$$

then $\omega$ is a twist point.

(The opposite implication holds as well, but will not be needed).

Proof. Let $\sigma$ be a curve in $D$ ending at $\omega$, that is to say, a continuous map $\sigma$ : $[0,1) \rightarrow D$ such that $\lim _{t \rightarrow 1} \sigma(t)=\omega$. Without loss of generality we may assume that $\sigma(0) \in D_{0}$. Let $\sigma^{*}:[0,1) \rightarrow \mathbb{R}$ be the continuous function (determined modulo a multiple of $2 \pi)$ such that $\sigma(t)-\omega=|\sigma(t)-\omega| e^{i \sigma^{*}(t)}$; we may also assume that $\left|\sigma^{*}(0)-\alpha\left(x_{0}\right)\right|<\pi / 2$ (thus uniquely determining $\left.\sigma^{*}\right)$. To prove the lemma, we show that for each curve $\sigma$ in $D$ ending at $\omega$, there is a sequence $\left\{t_{n}\right\} \subset[0,1)$ such that $\lim _{n \rightarrow \infty} t_{n}=1$ and

$$
\sigma^{*}\left(t_{n}\right)=\alpha(\omega(n))+\epsilon_{n}
$$


with $\left|\epsilon_{n}\right| \leq \pi / 2$. For $n=0$ and $t_{0}=0$ this statement follows from the definition of $\sigma^{*}$. For each $x \in T$ let $\Delta(x)$ be the equilateral triangle based on $x$ and pointing to the inside of $D$. Now let $t_{n}$ be the smallest $t$ such that $\sigma\left(t_{n}\right) \in \overline{\Delta(\omega(n))}$. It follows that $\sigma\left(t_{n}\right)$ also belongs to $\overline{\Delta(\omega(n-1))}$. Observe that for every $s \in \overline{\Delta(\omega(n))}$, the vector from $\omega$ to $s$ lies in a $\pi / 2$-cone around the inward normal vector to the basis of the triangle. So we have

$$
\sigma^{*}\left(t_{n}\right)=\alpha(\omega(n-1))+2 \pi k+\epsilon^{\prime}
$$

with $\left|\epsilon^{\prime}\right| \leq \pi / 2$ and

$$
\sigma^{*}\left(t_{n}\right)=\alpha(\omega(n))+2 \pi l+\epsilon^{\prime \prime}
$$

with $\left|\epsilon^{\prime \prime}\right| \leq \pi / 2$. Let us also assume that we already have proven that

$$
\sigma^{*}\left(t_{n-1}\right)=\alpha(\omega(n-1))+\epsilon_{n-1}
$$

with $\left|\epsilon_{n-1}\right| \leq \pi / 2$.

Observe that whenever the segment between $\sigma(s)$ and $\sigma(t)$ is contained inside $D$, then $\left|\sigma^{*}(s)-\sigma^{*}(t)\right|<\pi$. In fact, if we complete the curve from $\sigma(s)$ to $\sigma(t)$ with the segment between them, then we obtain a closed curve inside the simply connected domain $D$. (Note that between the times $s$ and $t$ the curve $\sigma$ might go anywhere in $D$ and $\sigma^{*}$ might become much greater or smaller). Now, the segment between $\sigma\left(t_{n-1}\right)$ and $\sigma\left(t_{n}\right)$ is contained in $D$. So we can deduce from (2) and (4) that $\left|2 \pi k+\epsilon^{\prime}-\epsilon_{n-1}\right|<\pi$, which implies $k=0$.

Since we also know that $|\alpha(\omega(n))-\alpha(\omega(n-1))| \leq \pi / 3$, we can conclude from (2) with $k=0$ and (3) that $\left|2 \pi l+\epsilon^{\prime \prime}-\epsilon^{\prime}\right| \leq \pi / 3$, which implies $l=0$. So finally we get (1) with $\epsilon_{n}:=\epsilon^{\prime \prime}$.

Proof of the theorem. By Lemma 3, it suffices to show that the following sets have harmonic measure zero:

$$
\begin{aligned}
& A_{+}:=\left\{\omega \in \Omega: \limsup _{n \rightarrow \infty} \alpha(\omega(n))=\infty \text { and } \liminf _{n \rightarrow \infty} \alpha(\omega(n))>-\infty\right\}, \\
& A_{-}:=\left\{\omega \in \Omega: \limsup _{n \rightarrow \infty} \alpha(\omega(n))<\infty \text { and } \liminf _{n \rightarrow \infty} \alpha(\omega(n))=-\infty\right\}, \\
& A_{d}:=\left\{\omega \in \Omega: \limsup _{n \rightarrow \infty} \alpha(\omega(n))-\liminf _{n \rightarrow \infty} \alpha(\omega(n))=d \pi / 3\right\} \text { for } d \in \mathbb{N} .
\end{aligned}
$$

Let us start with $A_{d}$ for some fixed $d \in \mathbb{N}$. Since the image of the function $\alpha$ is a discrete set, for each $\omega \in A_{d}$ there exists a number $n_{0}$ such that $\alpha\left(\omega\left(n_{0}\right)\right) \leq$ $\alpha(\omega(n)) \leq \alpha\left(\omega\left(n_{0}\right)\right)+d \pi / 3$ for all $n>n_{0}$. Therefore $A_{d}$ is a subset of $\bigcup_{x \in T} E_{d}^{\prime}(x)$,

$$
E_{d}^{\prime}(x):=\{\omega \in \Omega \cap E(x): \alpha(x) \leq \alpha(\omega(n)) \leq \alpha(x)+d \pi / 3 \text { for all } n \geq|x|\} .
$$

Since $T$ is countable, it suffices to show that $\nu\left(E_{d}^{\prime}(x)\right)=0$ for all $x \in T$. Define $\lambda_{+}^{1}(x):=\lambda_{+}(x)$, and, recursively, $\lambda_{+}^{n+1}(x):=\bigcup_{y \in \lambda_{+}^{n}(x)} \lambda_{+}(y)$. For each $y \in\{x\} \cup$ $\bigcup_{n=1}^{\infty} \lambda_{+}^{n}(x)$ there is a unique $\hat{y} \in \lambda_{+}^{d+1}(y)$ such that $\alpha(\hat{y})=\alpha(y)-(d+1) \pi / 3$; thus

$$
E_{d}^{\prime}(x) \cap E(y) \subset \bigcup_{z \in \lambda_{+}^{d+1}(y) \backslash\{\hat{y}\}} E(z) .
$$

In particular, we get

$$
E_{d}^{\prime}(x) \subseteq \bigcup_{x_{1} \in \lambda_{+}^{d+1}(x) \backslash\{\hat{x}\}} E\left(x_{1}\right)
$$


and therefore

$$
\nu\left(E_{d}^{\prime}(x)\right) \leq \sum_{x_{1} \in \lambda_{+}^{d+1}(x) \backslash\{\hat{x}\}} \nu\left(E\left(x_{1}\right)\right) \leq\left(1-\epsilon^{d+1}\right) \nu(E(x)),
$$

since, by Lemma $1, \nu(E(\hat{x})) \geq \epsilon^{d+1} \nu(E(x))$. Now we apply (5) in (6) and get

$$
E_{d}^{\prime}(x) \subseteq \bigcup_{x_{1} \in \lambda_{+}^{d+1}(x) \backslash\{\hat{x}\}} E\left(x_{1}\right) \cap E_{d}^{\prime}(x) \subseteq \bigcup_{\substack{x_{1} \in \lambda_{+}^{d+1}(x) \\ x_{1} \neq \hat{x}}} \bigcup_{\substack{x_{2} \in \lambda_{+}^{d+1}\left(x_{1}\right) \\ x_{2} \neq \hat{x}_{1}}} E\left(x_{2}\right)
$$

and therefore, applying Lemma 1 twice,

$$
\nu\left(E_{d}^{\prime}(x)\right) \leq\left(1-\epsilon^{d+1}\right)^{2} \nu(E(x)) .
$$

An iteration of these arguments gives $\nu\left(E_{d}^{\prime}(x)\right) \leq\left(1-\epsilon^{d+1}\right)^{n} \nu(E(x))$ for all $n$. Since $\left|1-\epsilon^{d+1}\right|<1$, it follows that $\nu\left(E_{d}^{\prime}(x)\right)=0$.

Finally, we show that $\nu\left(A_{+}\right)=\nu\left(A_{-}\right)=0$. Since $\pi^{-1}\left(J\left(A_{+}\right)\right)$and $\pi^{-1}\left(J\left(A_{-}\right)\right)$ are invariant under the shift and disjoint, and $\mu$ is ergodic, at least one of them has $\mu$-measure zero; then at least one of the sets $A_{+}$and $A_{-}$has zero $\nu$-measure, since $\mu\left(\pi^{-1}\left(J\left(A_{ \pm}\right)\right)\right.$is comparable to $\nu\left(A_{ \pm}\right)$, by Lemma 2 . On the other hand, the reflection on the line through $p$ perpendicular to $x_{0}$ exchanges $A_{+}$with $A_{-}$, leaving $\Omega$ and $\nu$ invariant; thus $\nu\left(A_{+}\right)=\nu\left(A_{-}\right)$. Thus $\nu\left(A_{ \pm}\right)=0$.

\section{REFERENCES}

[C85] L. Carleson, On the support of harmonic measure for sets of Cantor type, Ann. Acad. Sci. Fenn. Ser. AI Math 10 (1985), 113-123. MR 87b:31002

[HK76] W.K. Hayman and P.B. Kennedy, Subharmonic functions, Academic Press, London, 1976. MR 57:665

[KW85] R. Kaufman and J.M. Wu, On the snowflake domain, Ark. Mat. 23 (1985), 177-183. MR 86m:31002

[PTW92] M.A. Picardello, M.H. Taibleson and W. Woess, Harmonic measure of the planar Cantor set from the viewpoint of graph theory, Discrete Math. 109 (1992), 193-202. MR 94f:31009

[P92] Ch. Pommerenke, Boundary behaviour of conformal maps, Springer-Verlag, Berlin Heidelberg New York, 1992. MR 95b:30008

[S94] Ya.G. Sinai, Topics in ergodic theory, Princeton University Press, Princeton, N.J., 1994. MR 95j:28017

[vK06] H. von Koch, Une méthode géométrique élémentaire pour l'étude de certain questions de la théorie des courbes planes, Acta Math. 30 (1906), 145-174.

(F. Di Biase and B. Fischer) Department of Mathematics, Princeton University, PrinceTON, NeW JeRSEY 08544

E-mail address: biase@math.princeton.edu

E-mail address: fischer@math.princeton.edu

Room 2C-254, Bell Labs, Lucent Technologies, 600 Mountain Avenue, Murray Hill, NEW JERSEY 07974

E-mail address: ruediger@research.bell-labs.com 